EPJ Web of Conferences 71, 00110 (2014)

DOI: $10.1051 /$ epjconf / 20147100110

(C) Owned by the authors, published by EDP Sciences, 2014

\title{
Interpretations of Quantum Mechanics
}

\author{
Peter Pickl ${ }^{1, a}$ \\ ${ }^{1}$ Mathematisches Institut \\ LMU München \\ Theresienstr. 39 \\ 80333 München
}

\begin{abstract}
The problems of modern physics are man made. The Copenhagen version of quantum mechanics is formulated in a vague prosaic way, inconsistencies and paradoxes are the price. New interpretations try to solve the problem, however a reformulation rather than an interpretation is needed. In this manuscript I will point out, where the Copenhagen formulation of quantum mechanics is flawed and how one can make sense out of it. Then I will show, that it is possible to give a precise formulation of quantum mechanics without losing its compelling ability in describing experiments.
\end{abstract}

\section{Introduction}

There was a time when the newspapers said that only twelve men understood the theory of relativity. I do not believe there ever was such a time. There might have been a time when only one man did, because he was the only guy who caught on, before he wrote his paper. But after people read the paper a lot of people understood the theory of relativity in some way or other, certainly more than twelve. On the other hand, I think I can safely say that nobody understands quantum mechanics. So do not take the lecture too seriously, feeling that you really have to understand in terms of some model what I am going to describe, but just relax and enjoy it. I am going to tell you what nature behaves like. If you will simply admit that maybe she does behave like this, you will find her a delightful, entrancing thing. Do not keep saying to yourself, if you can possible avoid it, "But how can it be like that?" because you will get 'down the drain', into a blind alley from which nobody has escaped. Nobody knows how it can be like that.

(Richard Feynman)

Quantum mechanics is an incredibly successful theory. The accuracy of the predictions are striking (see for example [10]) and it has led to many remarkable technical innovations. But quantum mechanics is different to other theories like relativity or Newtonian mechanics. Feynman claims it is impossible to understand it. There are many similar remarks about quantum mechanics from other famous scientists and it not surprising that students usually find it very difficult to grasp the basic principles of the theory. However, I have to disagree with Feynman's statement. It is possible to get a

\footnotetext{
ae-mail: pickl@math.lmu.de
} 


\section{EPJ Web of Conferences}

picture from quantum mechanics which is as clear as Newtonian mechanics or relativity. In this paper I wish to explain the core of the problems and how some of the so called "interpretations of quantum mechanics" can solve them. The motivation for this manuscript is not to give an overview presenting all different "interpretations" (this has been done in other papers before) but to explain, why the usual way quantum mechanics is presented leads to confusions and how this can be avoided. Exemplarily I will present one formulation in more details [3] to show that it is in fact possible to present quantum mechanics in a mathematically precise and thus very clear way, although there are other qualified examples [11].

The central thesis of this paper will be, that the problems of quantum mechanics are caused by the fact, that it is usually formulated in an mathematically imprecise way. After the Copenhagen interpretation had been formulated, this was criticized by many contemporary scientists, the most prominent example being Albert Einstein. Unfortunately his points of criticism are neither understood nor taken serious by most physicists. Accompanied by the claim, that he did not understand quantum mechanics, they are usually ignored.

The problems of modern physics are caused by cultural misconducts of the recent years. Mathematics and physics have more and more been brought apart. Mathematicians are more and more interested in abstract issues irrelevant for our lives while physicists became more and more pragmatic, caring less about understanding than applications. The need of a precise formulation of the foundational issues is not taken serious enough.

The price one has to pay are paradoxes and philosophical problems. The distinction between physics and mysticism has started to erode: discussions of the role of consciousness etc. in connection to the foundational questions of quantum mechanics are not unusual. Consequently most physicists seek their asylum in that part of the theory, which is precise in clear: discussing the properties and behavior of the wave function. The few scientist who are interested in foundations usually try to solve these problems via interpretations. However no discussion about how an imprecise object should be interpreted makes the object precise! What one needs is a renaissance of a mathematical formulation of physics.

Compared to all other physical theories, quantum mechanics has caused the greatest shock of our world view. It is often claimed, that this change in our view of the world is necessary and, given the experimental facts, a mathematical precise formulation is impossible. This, however, is untrue!

The solution we shall present will, however, be less drastic than one might think, having read the lines above. The success of quantum mechanics is out of question. Having found the relevant properties of the wave function, comparisons with experimental data is possible and this is done with great success. Hence the wave function will be left untouched!

The task of this paper is very difficult. The usual formulations of quantum mechanics are accepted by most scientists. Growing up in contemporary culture, it is not easy to understand the necessity of a mathematical formulation of the foundations. Even worse: it is not easy to grasp the difference between a precise and an imprecise theory. Explaining a technical or experimental result is of course much easier than making head against the cultural misconduct of the last decades.

This paper is roughly organized in the following way: I will recall the most common formulation of quantum mechanics, the Copenhagen interpretation, and show that it is both imprecise and inconsistent. After that I wish to explain that understanding the Copenhagen interpretation as a effective rather than a fundamental theory, all the problems can be solved and the great success of the theory in its predictions can be understood. Finally I will introduce to Bohmian mechanics and respond to the usual criticism of the theory. I will explain, how Bohm's formulation of quantum mechanics can be used as a fundamental theory to derive all the statements of the Copenhagen interpretation. 


\section{The Copenhagen Interpretation}

If you can't explain it simply, you don't understand it well enough. (Albert Eintein)

To explain the reasons for the difficulties to understand quantum mechanics I will present the most prominent version taught at universities, the so called Copenhagen interpretation of quantum mechanics. The formulation of the theory is rather complicated, this is one of the reasons why Einstein never accepted the theory. The principles of the Copenhagen interpretation are as follows: [14]

1)A system is completely described by a wave function $\Psi$, representing the state of the system, which evolves smoothly in time, except when a measurement is made, at which point it instantaneously collapses to an eigenstate of the observable that is measured.

2)The description of nature is essentially probabilistic, with the probability of a given outcome of a measurement given by the square of the modulus of the amplitude of the wave function. (The Born rule, after Max Born)

3)It is not possible to know the value of all the properties of the system at the same time; those properties that are not known exactly must be described by probabilities. (Heisenberg's uncertainty principle)

4) Matter exhibits a wave-particle duality. An experiment can show the particle-like properties of matter, or the wave-like properties; in some experiments both of these complementary viewpoints must be invoked to explain the results, according to the complementarity principle of Niels Bohr.

5)Measuring devices are essentially classical devices, and measure only classical properties such as position and momentum.

6) The quantum mechanical description of large systems will closely approximate the classical description. (This is the correspondence principle of Bohr and Heisenberg.)

In addition to these "postulates" one has to define the Hilbert space in which the wave function $\Psi$ is located and the evolution equation for $\Psi$. Prominent examples are the space of square integrable functions on $\mathbb{R}^{3}$ or Fock space, respectively Schrödinger or Dirac equation in first or second quantization.

In this paper, the type of Hilbert space or evolution equation for $\Psi$ will be irrelevant! Hence the problems we address here are there for any quantum mechanical theory. To keep the paper as simple as possible, the solutions and examples shall solemnly given for the Schrödinger equation in first quantization.

\subsection{Formulating physical theories}

Philosophy is written in that great book which ever is before our eyes - I mean the universe - but we cannot understand it if we do not first learn the language and grasp the symbols in which it is written. The book is written in mathematical language, and the symbols are triangles, circles and other geometrical figures, without whose help it is impossible to comprehend a single word of it; without which one wanders in vain through a dark labyrinth.

(Galileo Galilei)

The central point of this paper is to show, that all the problems and difficulties of the way quantum theories are usually formulated are man made. Nature is fine! Also on atomic scales it behaves in 
a logical way, following mathematical rules. The formulation of quantum mechanics given above is erroneous. So what is the difference between the way physical theories were formulated before and after quantum mechanics?

More than 2000 years ago, Euclid formulated the fundamental principles of geometry in a mathematical language. The goal of Euclid was merely to understand the space surrounding us, not to give an abstract structure that can be used for different geometries. Thus euclidian geometry should be understood as one of the first physical theories. Euclids theory is formulated in different definitions, axioms and postulates. They were the fundament of his description of nature and formed the basis for a vast number of conclusions about space.

Although his formulation of geometry was slightly flawed - a corrected version was much later given by David Hilbert - Euclid understood that the formulation of a physical theory should be made with precision. Furthermore

- it should in principle be possible to derive all features of the world to be described from the fundamental principles

- the formulation of the theory should be irreducible, i.e. none of the fundamental principles must be derivable from the others.

Since then, physical theories have always been formulated according to these standards. Such a formulation is the basis for a clear and fundamental understanding of the physical model and makes ambiguities impossible. It has always been the vital characteristics of a physical theory. Like Galilei, physicists were convinced that mathematical language has to be used when talking about nature.

In addition to that, there has been the attempt to formulate the fundamental principles of the theory as simple as possible. After the axioms of euclidian geometry had been formulated, scientists tried to avoid the parallel postulate by proving it from the other axioms. The parallel postulate seemed less obvious than the others and, although its formulation is precise and clear, scientists tried to do without it for many centuries.

The credo that simple formulations of the principles should be searched for is supported by the great success of the principle of relativity.

\subsection{The formulation of the Copenhagen interpretation}

The violent reaction on the recent development of modern physics can only be understood when one realises that here the foundations of physics have started moving; and that this motion has caused the feeling that the ground would be cut from science.

(Werner Heisenberg)

It is often said, that the difficulties in understanding quantum mechanics is due to the fact that the phenomena the theory describes take place on scales which are different from our day to day experience. Evolution and experience have trained our senses to be able to cope with dimension relevant for our daily lives, excluding subatomic scales. But similar arguments are also true for relativity, which does not come with all these problems. But what is the difference between those two theories?

In contrast to Newtonian mechanics or relativity, some of the relevant elements of the theory are not defined in a precise way. While the wave function is given with mathematical preciseness, there is neither explanation of the words "experiment" and "measurement" nor when the experimenter should expect particle like properties and when he should expect wave like properties.

In contrast to this, classical mechanics is given by a evolution equation of a point in phase space. All ingredients of the theory are defined with mathematical rigor. 
This impreciseness in formulating the theory is the reason for all the problems, paradoxes and philosophical ballast, quantum mechanics is connected with.

One might argue, that it is clear, what a measurement apparatus is. But here one has to be careful! Clear in what sense? In the sense, that one can give a precise mathematical definition, or clear that for all practical purposes one can identify the measurement devices, i.e. in a sense as it is clear what a "car" is? In many practical situations, a vague understanding might be suffidient. But if one wants to get an unambiguous picture also at the crossover and wants to understand how a car (measurement device) works and why it drives (measures a property) it is not sufficient to name it.

There are also other deficits with the formulation of the theory, most of which are connected to or consequences of the impreciseness, for example:

a) Due to the vague formulation of the theory, one has to use intuition and experience rather than logic to judge, how the theory has to be used. For many practical purposes it might be clear when

- a many particle system should be treated quantum mechanically and when it has to be treated as a measurement device

- the wave function collapses

- the particle properties and when the wave properties have to be used.

However, there is no general rule and how to deal with the crossover between classical and quantum mechanical situations is unclear.

In an even worse manner this judgment may be different from different perspectives. Let us give an example: Assume one has some device in a black box that can be used for measuring the position of a photon of high energy. According to the theory the photon has to be treated quantum mechanically, the black box is a classical measurement apparatus. Now you open the box and find out, that it makes use of the Compton effect and finally measures the position of an electron.

What has played the role of the measurement device before and been treated classically has no to be explained using quantum mechanics.

b)Some of the "principles" of the theory aren't principles! This is obvious for "principle" 6). Proving that large systems behave classically is standard. "Postulate" 6) is not a postulate, it is a theorem! Also uncertainty is a direct consequence of a wave dynamics.

According to principle 1) it is only possible to measure the eigenvalues of observables. How can this be a principle? If there exists a way to build an apparatus which leads to perfect decoherence of different parts of the wave function which are not related to an eigenvalue of some operator, then this statement is wrong. If such a behavior is forbidden by the Schrödinger time evolution, then the statement is right but not a principle, then it is also a theorem!

c) The theory contradicts the principle of universality. Understanding the movement of planets and the trajectory of a thrown object as different aspects of the same fundamental principle has lead to Newtons formulation of gravity. In many different branches of physics one tries to understand different aspects of a theory by an underlying principle they have in common. The formulation of quantum mechanics distinguishes between the "system" described by the wave function and measurement devices. Their role entirely different.

Also in a Newtonian universe it is possible to talk about measurements and measurement devices. However such measurement devices have to be understood as special points in phase space and they are subject to the same evolution equation as all other particles. Consequently, for a classical universe "it is the theory which decides what can be observed" (Albert Einstein). 
d) With the problems above at hand it is not surprising, that quantum mechanics comes with all these paradoxes and philosophical ballast which is nonexistent in classical mechanics and relativity. We are all familiar with Schrödinger cat, Wigner's friend etc.

\subsection{Consequences}

There is no quantum world. There is only an abstract quantum physical description. It is wrong to think that the task of physics is to find out how nature is. Physics concerns what we can say about nature

(Niels Bohr)

It would seem that the theory [quantum mechanics] is exclusively concerned about "results of measurement", and has nothing to say about anything else. What exactly qualifies some physical systems to play the role of "measurer"? Was the wavefunction of the world waiting to jump for thousands of millions of years until a single-celled living creature appeared? Or did it have to wait a little longer, for some better qualified system ... with a Ph.D.? If the theory is to apply to anything but highly idealized laboratory operations, are we not obliged to admit that more or less "measurement-like" processes are going on more or less all the time, more or less everywhere. Do we not have jumping then all the time?

(John Bell)

Using the standards Euclid has applied to his formulation of geometry and that was valid for many centuries, the Copenhagen interpretation is not a physical theory. It is not formulated in a precise way, it is not written "in mathematical language", its symbols are not "triangles, circles and other geometrical figures". While the "wave function" is a mathematical object, the word "measurement" is never defined and is not trivial to do so. In addition to that, the "principles" are not irreducible!

Nevertheless it is accepted by most physicist and comparably little effort is being made to reformulate it. More than two thousand years scientist have concentrated all their efforts in reformulating or deducting the parallel axiom of euclidian geometry, although the theory was precise and clear, just to cure a merely cosmetic problem of a flawless theory. In contrast to that little effort has been spent to improve the formalism of quantum mechanics, although it is an imprecise mixture of principles and consequences. Although there was criticism of the theory by as prominent members of the scientific community like Albert Einstein, only few physicists tried to improve its formulation.

Instead of adjusting the formulation of the theory according to the centuries-old standards about how a physical theory should in principle look like, the idea of how physics should be has changed. For example the position of Niels Bohr quoted above was not born out of a philosophical necessity. This position was introduced to justify the formulation of the theory.

\subsection{Making sense out of the Copenhagen interpretation}

I have the greatest consideration for the goals which are pursued by the physicists of the latest generation which go under the name of quantum mechanics, and I believe that this theory represents a profound level of truth, but I also believe that the restriction to laws of a statistical nature will turn out to be transitory.... Without doubt quantum mechanics has grasped an important fragment of the truth and will be a paragon for all future fundamental theories, for the fact that it must be deducible as a limiting case from such foundations, just as electrostatics is deducible from Maxwell's equations of the 
electromagnetic field or as thermodynamics is deducible from statistical mechanics. (Albert Einstein)

As explained above, the Copenhagen interpretation can not be understood as a fundamental physical theory. On the other hand there is a lot of truth in its statements. Without any doubt the predictions of the theory are in outstanding agreement with experiments.

To solve the problems mentioned above, it is important to be more precise, in particular the meaning of the word "measurement" has to be clarified. A precise theory neither needs an interpretation nor a philosophical addendum. All paradoxes can be resolved, their answers lie in the equations.

So how can the notion "measurement" be made precise?

In any case, a measurement always includes an interaction with a macroscopic system, i.e. the measurement device or the observer. In a universal quantum mechanical theory a macroscopic object can only be understood in an idealized situation. Obviously all measurement devices are part of our physical world and thus made out of quantum mechanical particles. However, in such a large number that for all practical purposes they can be treated as classical objects.

Consequently the Copenhagen interpretation can only make sense if one understands it as an effective theory: A measurement has to be defined as an interaction with an apparatus in an idealized situation, which leads to perfect decoherence. Having perfect decoherence of different parts of the wave functions, their time evolution can be treated separately and a collapse can be introduced without changing the time evolution of the system.

Hence the Copenhagen interpretation is not meant to give a description of our universe as it is, it describes an universe which is idealized in some of its aspects. Only in some limit which assures perfect decoherence after the measurement process, the two theories match. Of course there is always some difference, but for all practical purposes this difference can be neglected.

In contrast to that, the Copenhagen interpretation is usually presented in textbooks and lectures as being a fundamental theory. The word "fundamental" should not be understood in the sense that there is no other underlying theory and that it describes all known physical phenomena. Here "fundamental" means that the theory itself needs no further explanation and can be understood without reference to an underlying microscopic theory. In this sense, Newtonian mechanics and relativity are both fundamental theories.

Hence the theory itself is not problematic, it is only presented in a wrong way.

Understanding Copenhagen interpretation as an effective theory, all the problems mentioned above are solved:

a) The dichotomies explained in a) are solved. Depending on how close we are to the idealized limiting regime, a classic description of some of the objects is legitimate. No crossover has to be understood.

b) An effective theory is not based on fundamental principles. All the "principles" mentioned above are consequences of the microscopic description (see below) and should be proven.

c) An effective theory naturally uses different descriptions for systems which are close to the idealized situations and systems which aren't. Of course the underlying fundamental equation should be universal.

d) Since the theory is not qualified to describe situations on the crossover, all paradoxes vanish. They can be understood in the respective microscopic theory.

Furthermore it is not surprising that the theory is so successful. With experience and intuition at hand, it is not problematic to judge, when one is close to the idealized situations. But intuition is used merely to avoid the effort of a technical analysis, in principle such a mathematical analysis is possible. An whenever there is a paradox or ambiguity, one can use the microscopic model to get a precise answer. 


\subsection{Can the Copenhagen interpretation be derived}

The idea of an objective real world whose smallest parts exist objectively in the same sense as stones or trees exist, independently of whether or not we observe them. . . is impossible. ...

(Werner Heisenberg)

Is a derivation as described by Einsteins quote in the beginning of section 2.4 desirable? Is it desirable to have a formulation of quantum mechanics in a mathematical language? Would it be nice to have a precise theory without the need of interpretation and philosophical ballast? A formulation in which the theory decides, what measurements are, where the collapse is a natural consequence of the dynamics that can be understood from the microscopic point of view and where the "principles" 1) to 6) are theorems?

The mindset of the physical community to that question has changed with the upcoming of quantum mechanics. Nowadays it is not exotic to find a physicists who would answer this question with "no". From a pragmatic point of view, such a microscopic derivation might be of little or no relevance. An effective theory is sufficient to compare theoretical predictions to result of measurements. However, the relevance of physics is the understanding of nature, more than the comparing of results of measurements. And a microscopic understanding is required to get rid of all the philosophical ballast and to resolve the paradoxes.

The second question is: is it possible to derive the Copenhagen version of quantum mechanics from a universal theory? Although most physicists share Heisenbergs view on quantum mechanics rather than Einsteins, the answer is "yes".

There is no room for discussion to this second answer. Heisenberg was proven wrong by giving examples of such theories!

\section{Bohmian mechanics}

Is it not clear from the smallness of the scintillation on the screen that we have to do with a particle? And is it not clear, from the diffraction and interference patterns, that the motion of the particle is directed by a wave? De Broglie showed in detail how the motion of a particle, passing through just one of two holes in a screen, could be influenced by waves propagating through both holes. And so influenced that the particle does not go where the waves cancel out, but is attracted to where they cooperate. This idea seems to me so natural and simple, to resolve the wave-particle dilemma in such a clear and ordinary way, that it is a great mystery to me that it was so generally ignored.

(John Bell)

One example of a theory which can be used to derive the Copenhagen interpretation of quantum mechanics is the so called "Bohmian mechanics". The principle idea of the theory goes back to de Broglie [4], who presented it at the Solvay conference 1927 and was later independently formulated by David Bohm [3]. Since it is more important to explain, how such a derivation can be done in principle, than to give an overview of all approaches known, I will work out solemnly that example in detail. I wish to not that the collapse model introduced by Ghirardi, Rimini and Weber [11] is a candidate which is qualified as well as Bohmian mechanics. Both, Bohmian mechanics and GRW are formulated with mathematical precision. Both do reproduce all quantum mechanical results in the non relativistic regime. Also relativistic versions could be formulated [5, 13]. 
There are other attempts, however I am skeptical with any approach that understands itself as an "interpretation" of quantum mechanics rather than a microscopic derivation and/or fundamentally relies on "measurements' or other words that are not defined precisely.

\subsection{The theory}

Conventional formulations of quantum theory, and of quantum field theory in particular, are unprofessionally vague and ambiguous. Professional theoretical physicists ought to be able to do better. Bohm has shown us a way.

(John Bell)

Compared to other quantum mechanical theories Bohmian mechanics is very simple. It is a theory about the time evolution of $N$ of particles. To keep the notation simple we assume that all magnetic fields are zero and that all particles have the same mass $m$. In units $\hbar=m=1$ the guiding law for the respective point $Q(t)=\left(q_{1}(t), \ldots, q_{N}(t)\right)$ in configuration space (here $q_{j}(t) \in \mathbb{R}^{3}$ is the position of the $j^{\text {th }}$ particle at time $t$ ) is then given by

$$
\frac{d Q}{d t}:=v^{\Psi}(Q)=\mathfrak{J} \frac{\Psi^{\star} \nabla \Psi}{\Psi \star \Psi}(Q)
$$

$\Psi$ is a solution of the $N$-particle Schrödinger equation

$$
i \frac{d \Psi}{d t}=-\sum_{j=1}^{N} \nabla_{j}^{2} \Psi+V \Psi .
$$

One further assumes that the initial configuration $Q(0)$ is typical with respect to the measure given by $|\Psi(0)|^{2}$. Given a wave function $\Psi\left(t_{0}\right)$ and a configuration $Q\left(t_{0}\right)$ it is possible to compute $\Psi(t)$ and $Q(t)$ for all times $t \in \mathbb{R}$, so the evolution equation is deterministic. The pair $\left(Q\left(t_{0}\right), \Psi\left(t_{0}\right)\right)$ is the state of a Bohmian system at time $t$.

In contrast to the Copenhagen interpretation, where $\Psi$ always describes subsystems of the universe excluding at least the measurement apparatus, the Bohmian formulation of quantum mechanics can be used for the universe as a whole as well as for subsystems that do not interact with the environment. As soon as the system interacts with the environment, that description has to be adjusted accordingly. How this is done shall be explained below.

Obviously Bohmian mechanics is mathematically precise. It is a theory about two mathematically well defined objects, the wave function $\Psi$ and the configuration $Q$. There is no need to talk about measurements to understand the theory. However it is possible - exactly like in Newtonian mechanics or relativity - to give names to certain configurations of particles, like "chair", "tree", "car" or "measurement apparatus" and to understand them from microscopic principles. Hence all measurement situations which we know from physical experiments of our daily lives are reflected in the respective configuration $Q$ which gives the positions of all the particles which form the measurement device and the measured particles.

Due to its precise formulation, Bohmian mechanics is easy to understand and can do without interpretations or philosophy. Furthermore it is possible to derive all the "principles" of the Copenhagen interpretation whenever we are in the idealized situation where the latter makes sense.

\subsection{Criticism}

Before criticizing a theory, one first has understand how and why the theory was formulated. It is often claimed, that the motivation of the "Bohmians" is to keep the idea of classical particles and/or to 
get rid of a probabilistic theory. This is untrue. The motivation is to formulate quantum mechanics in mathematical language. The fact that this formulation uses the idea of particles and is a deterministic theory is unimportant. Another precise formulations of quantum mechanics, the collapse models developed by Ghirardi, Rimini and Weber [11] is esteemed in the Bohmian community. Such collapse models are probabilistic theories and not about particles in the classical sense.

The role of Bohmian mechanics is not to give any new experimental data. In contrary, the goal is to derive the Copenhagen version of quantum mechanics and hence to give exactly the same predictions as the Copenhagen version of quantum mechanics whenever the latter makes sense. Any criticism that Bohmian mechanics does not give any new experimental data is pointless.

Bohmian mechanics is often criticized for its requirement of an additional equation (1). But any theory describing quantum mechanics needs something additional to the wave function! The wave function alone can not explain the occurrence of spots on a screen, it does not lead to duality etc.. In the Copenhagen interpretation the additives are the "principles" 1) - 6). Why should one prefer as much as six prosaic, imprecise principles to one mathematical formula?

It is widely believed, that hidden variable theories like Bohmian mechanics are impossible. However all the no-go theorems require additional assumptions and do not rule Bohmian mechanics. Bell [2] showed for example the the proof of von Neumann [12] is based on assumptions that are not satisfied by Bohmian mechanics. Trying to find a no-go theorem which excludes Bohmian mechanics is futile: since it has been proven that the Bohmian and the Copenhagian predictions match, one can only rule out both or none of these theories!

One should also note that, although finding a Lorentz invariant formulation of the theory is not trivial, Bohmian versions of field theories are possible [5].

\section{Deriving the "principles" of the Copenhagen interpretation}

For those who agree that a mathematical precise formulation of quantum mechanics is desirable and understand, that the Copenhagen interpretation is not qualified, we have come to the interesting point of this paper. The derivation of the "principles" 1)-6) from Bohmian mechanics. In this paper I will concentrate on the Born rule and the collapse of the wave function.

\subsection{Born's rule}

As we have explained above, one assumes that the initial configuration $Q_{0}$ is typical with respect to $|\Psi(0)|^{2}$. This is the so called quantum equilibrium hypotheses (see also [6, 7]). Since the Bohmian trajectories are the flow lines of the $\left|\Psi^{2}\right|$ fields it follows that for any time $t$ the distribution of the configuration $Q(t)$ is typical with respect to $|\Psi(t)|^{2}$. Consequently, the probability that the configuration is in some subset $\mathcal{A}$ of configuration space is given by $\int_{\mathcal{A}}|\Psi(x)|^{2} d^{3 N} x$.

Note that any measurement will finally lead to a decoherent split-up of the wave-function in configuration space, coming from the different positions of the particles forming the pointer of the apparatus or the positions of the photons coming from the screen of the device. The probability that a pointer will indicate a specific result is given by the measure of all the respective trajectories, i.e. the modulus square of the respective part of the wave function. Thus the Born rule is a consequence of the continuity equation.

\subsection{Measurements and the collapse of the wave function}

In the Copenhagen interpretation a measurement changes the physical description of a system. 
In Bohmian mechanics a measurement is precisely what a it should be: it is nothing more than an update of the information that we have of the system! Given the fact that a pointer (or photons coming from a display etc.) of a measurement apparatus points into a certain direction we can draw conclusions on the configuration of the system. Before the measurement we had to describe our system via the different probabilities of all the possible configurations, being ignorant about the true configuration of the system. After the measurement we can do better and describe the system under the condition that the measurement has given us a certain outcome. Consequently the number of possible configurations has dropped, the probabilities of the remaining configurations is given by the conditional probabilities, given that the pointer points into a certain direction. Now the dynamics of the remaining configurations can be described by a new wave function, the so called conditional wave function. One can show that in situation where an observable is measured, this conditional wave function is a eigenfunction of the observable. In Bohmian mechanics the collapse of the wave function is merely an adjustment of the description of our system according to the information we got from the measurement.

Let us explain: consider a physical system that consists of a subsystem and an environment and that the initial wave function $\Psi\left(t_{0}\right)=\phi\left(t_{0}\right) \chi\left(t_{0}\right)$ is a product of a wave function $\phi\left(t_{0}\right)$ describing the subsystem and a wave function $\chi\left(t_{0}\right)$ describing the environment.

We assume that the interaction between subsystem and environment is negligible for times $t<t_{i}$ and times $t>t_{f}$, where $t_{0}<t_{i}<t_{f}$.

Such a systems is well suited to describe a measurement situation: the subsystem plays the role of the particle which is measured, the environment includes the measurement device. Both will only interact during a certain time interval, when the particle is under the influence of the apparatus.

Assume that our measurement can lead to a finite number of different outcomes collected in a set $O$. Due to the interaction, $\Psi(t)$ is in general not a product after the measurement has been made, for times $t>t_{f}$ it can be written as a sum

$$
\Psi(t)=\sum_{j \in O} \phi^{j}(t) \chi^{j}(t) .
$$

Since the $\chi^{j}$ stand for different pointer positions of the apparatus, the wave functions $\chi^{j}$ are pairwise spatially separated. Assume that the outcome of the measurement is "o", i.e. the pointer points in the respective direction. Then only those configuration, where the particles forming the pointer are in the support of $\chi^{\circ}$ are relevant for our further description.

Due to decoherence of the different summands of (3) one can use $\phi^{o} \chi^{o}$ to describe the time evolution of the possible configurations that are left in very good approximation. The other parts of the wave function $\phi^{j} \chi^{j}$ for $j \neq o$ are in the idealized situation of perfect decoherence irrelevant for the time evolution. Hence the collapse is an effective collapse of the wave function. Both, the full and the collapsed wave function describe the time evolution of the trajectories. The descriptions match exactly in the limit where the apparatus leads to perfect decoherence. For all practical purposes, the error coming from the reduction of the wave function ot an eigenstate is negligible.

Hence the collapse is a mathematical consequence of the adjustment of the description of the system according to the experimental data. It is not put into the theory by brute force.

A more detailed explanation of Bohmian mechanics and how it can be used to explain the quantum mechanical predictions with mathematical rigor can be found in textbooks [8,9]. Many details and further readings on Bohmian mechanics are given on the website www.bohmian-mechanics.net. In its explanations, how the collapse of the wave function comes up naturally in Bohmian mechanics, a talk of Stefan Teufel that can be found on youtube is very descriptive. 


\section{References}

[1] G. Bacciagaluppi, A. Valentini: Quantum Theory at the Crossroads, (Cambridge University Press 2009)

[2] J. S. Bell: On the problem of hidden variables in quantum mechanics. Rev. Mod. Phys. 38, 447-452 (1966);

[3] D. Bohm: A Suggested Interpretation of the Quantum Theory in Terms of "Hidden" Variables, I and II. Phys. Rev. 85, 166-193 (1952)

[4] L. de Broglie: In Electrons et Photons: Rapports et Discussions du Cinquième Conseil de Physique tenu à Bruxelles du 24 au 29 Octobre 1927 sous les Auspices de l'Institut International de Physique Solvay, (Gauthier-Villars, Paris 1928); English translation in [1].

[5] D. Dürr, S. Goldstein, T. Norsen, W. Struyve, N. Zanghì: Can Bohmian mechanics be made relativistic?, Proc. R. Soc. A 470, 20130699 (2014)

[6] D. Dürr, S. Goldstein, N. Zanghì: it Quantum Equilibrium and the Origin of Absolute Uncertainty., J. Stat. Phys. 67, 843-907 (1992)

[7] D. Dürr, S. Goldstein, N. Zanghì: Quantum Equilibrium and the Role of Operators as Observables in Quantum Theory., J. Stat. Phys. 116, 959-1055 (2004)

[8] D. Dürr, s. Goldstein, N. N. Zanghì:: Quantum Physics Without Quantum Philosophy, Springer, Berlin (2012)

[9] D. Dürr, S. Teufel: Bohmian Mechanics, Springer, Berlin (2009)

[10] G. Gabrielse, D. Hanneke, T. Kinoshita, M. Nio and B. Odom: New Determination of the Fine Structure Constant from the Electron $g$ Value and QED, Phys. Rev. Lett. 97, 030802 (2006)

[11] Ghirardi, G.C., Rimini, A., and Weber, T.: Unified dynamics for microscopic and macroscopic systems,

[12] von Neumann, J: Mathematische Grundlagen der Quantenmechanik, Springer, Berlin, (1932)

[13] Tumulka, R.: A Relativistic Version of the Ghirardi-Rimini-Weber Model, J. Statist. Phys. 125 821-840 (2006)

[14] http://en.wikipedia.org/wiki/Copenhagen_interpretation (08.01.2014) 